\title{
Existence of solutions for fractional differential equations with infinite point boundary conditions at resonance
}

Wei Zhang and Wenbin Liu*

"Correspondence:

liuwenbin-xz@163.com School of Mathematics, China

University of Mining and

Technology, Xuzhou, P.R. China

\begin{abstract}
In this paper, by using Mawhin's continuation theorem, we establish some sufficient conditions for the existence of at least one solution for a class of fractional infinite point boundary value problem at resonance. Moreover, an example is given to illustrate our results.
\end{abstract}

MSC: 34A08; 34B15

Keywords: Fractional differential equation; Infinite point boundary value problem; Resonance; Mawhin's continuation theorem

\section{Introduction}

In the past 20 years, fractional differential equations (FDEs for short) as mathematical models have been successfully used in many fields of science and engineering (see [1-7]), which have attracted considerable attention in studying FDEs. For example, Izhikevich neuron model can be described by two fractional-order differential equations as the form

$$
\left\{\begin{array}{l}
\tau^{C} D^{\alpha} v(t)=f v^{2}+g v+h-u+R I, \\
\tau^{C} D^{\alpha} u(t)=a(b v-u) I,
\end{array}\right.
$$

where $\alpha \in(0,1],{ }^{C} D^{\alpha}$ is the Caputo fractional derivative of order $\alpha, v(t)$ represents the membrane voltage and $u(t)$ express the recovery variable (see [6]). Ates and Zegeling [7] studied the following fractional-order advection-diffusion reaction boundary value problems (BVPs for short):

$$
\left\{\begin{array}{l}
\varepsilon^{C} D^{\alpha} u+\gamma u^{\prime}+f(u)=S(x), \quad x \in[0,1], \\
u(0)=u_{L}, \quad u(1)=u_{R},
\end{array}\right.
$$

where $1<\alpha \leq 2,0<\varepsilon \leq 1, \gamma \in \mathbb{R},{ }^{C} D^{\alpha}$ is the Caputo fractional derivative of order $\alpha$. The function $S(x)$ represents a spatially dependent source term.

Recently, fractional differential equations with various kinds of boundary conditions (BCs for short) have been discussed widely and obtained numerous valuable results (see

(c) The Author(s) 2018. This article is distributed under the terms of the Creative Commons Attribution 4.0 International License (http://creativecommons.org/licenses/by/4.0/), which permits unrestricted use, distribution, and reproduction in any medium, provided you give appropriate credit to the original author(s) and the source, provide a link to the Creative Commons license, and indicate if changes were made. 
[8-31]). It is worth mentioning that the discuss of fractional differential equations with infinite point BCs have been attracted many scholars' attention over the past two years (see [18-31]). These work studied on many subjects, such as: existence of solutions, positive solutions, multiple solutions, unique solution. In $[18,19]$ Zhang and Zhai et al. considered the following fractional differential equation with infinite point BCs:

$$
\left\{\begin{array}{l}
D_{0+}^{\alpha} u(t)+q(t) f(t, x(t))=0, \quad t \in(0,1) \\
u(0)=u^{\prime}(0)=\cdots=u^{(n-2)}(0)=0 \\
u^{(i)}(1)=\sum_{j=1}^{\infty} \alpha_{j} u\left(\xi_{j}\right)
\end{array}\right.
$$

respectively, where $\alpha>2, n-1<\alpha \leq n, i \in[1, n-2]$ is a fixed integer, $\alpha_{j} \geq 0,0<\xi_{1}<$ $\xi_{2}<\cdots<\xi_{j-1}<\xi_{j}<\cdots<1(j=1,2 \ldots), D_{0+}^{\alpha}$ is the standard Riemann-Liouville fractional derivative of order $\alpha$. By employing the fixed-point theorem in cones, Zhang established the existence and multiplicity of positive solutions theorems and Zhai et al. obtained the existence and uniqueness result on positive solutions.

In [20], Guo et al. investigated the following infinite point fractional BVPs:

$$
\left\{\begin{array}{l}
{ }^{C} D_{0+}^{\alpha} u(t)+f\left(t, u(t), u^{\prime}(t)\right)=0, \quad 0<t<1, \\
u(0)=u^{\prime \prime}(0)=0, \quad u^{\prime}(1)=\sum_{j=1}^{\infty} \eta_{j} u\left(\xi_{j}\right),
\end{array}\right.
$$

where $2<\alpha \leq 3, \eta_{j} \geq 0,0<\xi_{1}<\xi_{2}<\cdots<\xi_{j-1}<\xi_{j}<\cdots<1(j=1,2, \ldots),{ }^{C} D_{0_{+}}^{\alpha}$ is the Caputo fractional derivative of order $\alpha$. The authors obtained the existence of multiple positive solutions by means of Avery-Peterson's fixed-point theorem.

Although many papers dealing with fractional infinite points BVPs, only a few papers consider FDEs with infinite point BCs at resonance (see [21-23]). In [21], Ge et al. discussed the following coupled FDEs with infinitely points BCs at resonance:

$$
\left\{\begin{array}{l}
D_{0+}^{\alpha} x_{1}(t)=f_{1}\left(t, x_{1}(t), D_{0+}^{\beta-1} x_{2}(t)\right), \\
D_{0+}^{\beta} x_{2}(t)=f_{2}\left(t, x_{2}(t), D_{0+}^{\alpha-1} x_{1}(t)\right), \\
x_{1}(0)=0, \quad \lim _{t \rightarrow \infty} D_{0+}^{\alpha-1} x_{1}(t)=\sum_{i=1}^{+\infty} \gamma_{i} x_{1}\left(\eta_{i}\right), \\
x_{2}(0)=0, \quad \lim _{t \rightarrow \infty} D_{0+}^{\beta-1} x_{2}(t)=\sum_{i=1}^{+\infty} \sigma_{i} x_{2}\left(\xi_{i}\right),
\end{array}\right.
$$

where $1<\alpha, \beta \leq 2,0<\eta_{1}<\eta_{2}<\cdots<\eta_{i}<\cdots, 0<\xi_{1}<\xi_{2}<\cdots<\xi_{i}<\cdots, \lim _{i \rightarrow \infty} \eta_{i}=\infty$, $\lim _{i \rightarrow \infty} \xi_{i}=\infty$ and $\sum_{i=1}^{+\infty}\left|\gamma_{i}\right| \eta_{i}^{\alpha}<\infty, \sum_{i=1}^{+\infty}\left|\sigma_{i}\right| \xi_{i}^{\beta}<\infty, D_{0+}^{\alpha}$ and $D_{0+}^{\beta}$ are standard RiemannLiouville fractional derivative. By using Mawhin's continuous theorem the authors obtained the existence result.

Thus, motivated by the results mentioned, the purpose of this paper is to present the existence of solutions for the following infinite point BVPs by applying Mawhin's continuous theorem:

$$
\left\{\begin{array}{l}
D_{0+}^{\alpha} x(t)=f\left(t, x(t), D_{0+}^{\alpha-2} x(t), D_{0+}^{\alpha-1} x(t)\right), \quad t \in(0,1), \\
x(0)=0, \quad D_{0+}^{\alpha-1} x(0)=\sum_{i=1}^{+\infty} \alpha_{i} D_{0+}^{\alpha-1} x\left(\xi_{i}\right), \\
D_{0+}^{\alpha-1} x(1)=\sum_{i=1}^{+\infty} \beta_{i} D_{0+}^{\alpha-1} x\left(\gamma_{i}\right)
\end{array}\right.
$$


where $2<\alpha \leq 3, D_{0+}^{\alpha}$ is the standard Riemann-Liouville fractional derivative of order $\alpha$, $f \in[0,1] \times \mathbb{R}^{3} \rightarrow \mathbb{R}$ is a Carathéodory function, $\xi_{i}, \gamma_{i} \in(0,1)$ and $\left\{\xi_{i}\right\}_{i=1}^{+\infty},\left\{\gamma_{i}\right\}_{i=1}^{+\infty}$ are two monotonic sequence with $\lim _{i \rightarrow+\infty} \xi_{i}=a, \lim _{i \rightarrow+\infty} \gamma_{i}=b, a, b \in(0,1), \alpha_{i}, \beta_{i} \in \mathbb{R}$.

Throughout this paper, we assume that the following condition holds:

$$
\begin{aligned}
\left(\mathrm{H}_{1}\right) \sum_{i=1}^{+\infty} \alpha_{i}=1, \sum_{i=1}^{+\infty} \beta_{i}=1, \sum_{i=1}^{+\infty}\left|\alpha_{i}\right|<+\infty, \sum_{i=1}^{+\infty}\left|\beta_{i}\right|<+\infty, \Delta \neq 0, \text { where } \\
\Delta=a_{11} a_{22}-a_{12} a_{21}, \quad a_{11}=\sum_{i=1}^{+\infty} \alpha_{i} \xi_{i}, \quad a_{12}=\sum_{i=1}^{+\infty} \alpha_{i} \xi_{i}^{2}, \\
a_{21}=1-\sum_{i=1}^{+\infty} \beta_{i} \gamma_{i}, \quad a_{22}=1-\sum_{i=1}^{+\infty} \beta_{i} \gamma_{i}^{2} .
\end{aligned}
$$

Remark 1.1 Here, if we let $\alpha_{i}, \beta_{i} \in \mathbb{R}^{+}, a^{2} \leq \xi_{1}, \gamma_{1}^{2} \leq b$ and assume that $\left\{\xi_{i}\right\}_{i=1}^{+\infty},\left\{\gamma_{i}\right\}_{i=1}^{+\infty}$ are monotonically increasing sequence and monotonically decreasing sequence, respectively, then, by $\left(\mathrm{H}_{1}\right)$, we have $\Delta>0$.

In fact, since $\left\{\xi_{i}\right\}_{i=1}^{+\infty}$ monotone increasing and $\left\{\gamma_{i}\right\}_{i=1}^{+\infty}$ monotone decreasing with $\lim _{i \rightarrow+\infty} \xi_{i}=a, \lim _{i \rightarrow+\infty} \gamma_{i}=b, a, b \in(0,1)$, then by $\left(\mathrm{H}_{1}\right)$ we have

$$
\begin{aligned}
\Delta & =a_{11} a_{22}-a_{12} a_{21} \\
& =\sum_{i=1}^{+\infty} \alpha_{i} \xi_{i}\left(1-\sum_{i=1}^{+\infty} \beta_{i} \gamma_{i}^{2}\right)-\sum_{i=1}^{+\infty} \alpha_{i} \xi_{i}^{2}\left(1-\sum_{i=1}^{+\infty} \beta_{i} \gamma_{i}\right) \\
& >\xi_{1}\left(1-\gamma_{1}^{2}\right)-a^{2}(1-b) \geq 0 .
\end{aligned}
$$

A boundary value problem is called resonance if the corresponding homogeneous boundary value problem has a nontrivial solution. We point out that if condition $\left(\mathrm{H}_{1}\right)$ holds, the BVP (1.1) happens to be at resonance in the sense that the following infinite point boundary value problem:

$$
\left\{\begin{array}{l}
D_{0+}^{\alpha} x(t)=0, \quad t \in(0,1) \\
x(0)=0, \quad D_{0+}^{\alpha-1} x(0)=\sum_{i=1}^{+\infty} \alpha_{i} D_{0+}^{\alpha-1} u\left(\xi_{i}\right) \\
D_{0+}^{\alpha-1} x(1)=\sum_{i=1}^{+\infty} \beta_{i} D_{0+}^{\alpha-1} x\left(\gamma_{i}\right)
\end{array}\right.
$$

has $x(t)=a_{1} t^{\alpha-1}+a_{2} t^{\alpha-2}, a_{1}, a_{2} \in \mathbb{R}$ as a nontrivial solution. That means the linear operator $L x=D_{0+}^{\alpha} x$ is non-invertible.

Even though in the papers [21,22] and [23] authors have investigated the resonance case with $\operatorname{dimKer} L=2$, all of them considered with the coupled fractional differential equations, in which the linear operator $L$ defined as $L(x, y)=\left(L_{1} x, L_{2} y\right)$ and $\operatorname{dimKer} L_{1}=$ $\operatorname{dimKer} L_{2}=1$. In our paper, we study the one fractional differential equation resonance case with $\operatorname{dim} \operatorname{Ker} L=2$, which is obviously different from papers [21, 22] and [23]. Compared with previous work the main difficulties in this paper are as follows. First, resonance BVPs cannot be dealt with fixed-point theorem directly. Second, the theory of Mawhin's continuation theorem is characterized by the higher dimension of kernel space on resonance BVPs, the more difficult to construct the projections $P$ and $Q$. Third, we give an example to support our main result, and we should point out that to give an example for BVPs (1.1) is very difficult. 
The rest of this paper is organized as follows. In Sect. 2, we recall some preliminary definitions and lemmas. In Sect. 3, based on Mawhin's continuation theorem, we establish an existence theorem for problem (1.1). In Sect. 4, we present an example to demonstrate the results. In the last section, brief conclusions are given.

\section{Preliminaries}

In this section, we recall some definitions and lemmas which are used throughout this paper. First, we present here the results of coincidence degree theory due to Mawhin which can be found in [32,33].

Let $\left(X,\|\cdot\|_{X}\right)$ and $\left(Y,\|\cdot\|_{Y}\right)$ are two real Banach spaces. Define $L: \operatorname{dom} L \subset X \rightarrow Y$ to be a Fredholm operator with index zero, then there exist two continuous projectors $P: X \rightarrow$ $X$ and $Q: Y \rightarrow Y$ such that

$$
\operatorname{Im} P=\operatorname{Ker} L, \quad \operatorname{Im} L=\operatorname{Ker} Q, \quad X=\operatorname{Ker} L \oplus \operatorname{Ker} P, \quad Y=\operatorname{Im} L \oplus \operatorname{Im} Q,
$$

and $\left.L\right|_{\operatorname{dom} L \cap \operatorname{Ker} P}: \operatorname{dom} L \rightarrow \operatorname{Im} L$ is invertible. We denote its inverse by $K_{p}$. Let $\Omega$ be an open bounded subset of $X$ and $\operatorname{dom} L \cap \bar{\Omega} \neq \emptyset$, then the map $N: X \rightarrow Y$ is called $L$-compact on $\bar{\Omega}$, if $Q N(\bar{\Omega})$ is bounded and $K_{P, Q} N=K_{p}(I-Q) N: \bar{\Omega} \rightarrow X$ is compact.

Theorem 2.1 Let $L: \operatorname{dom} L \subset X \rightarrow Y$ be a Fredholm operator of index zero and $N: X \rightarrow Y$ be L-compact on $\bar{\Omega}$. Assume that the following conditions are satisfied:

(i) $L u \neq \lambda N u$ for any $u \in(\operatorname{dom} L \backslash \operatorname{Ker} L) \cap \partial \Omega, \lambda \in(0,1)$;

(ii) $N u \notin \operatorname{Im} L$ for any $u \in \operatorname{Ker} L \cap \partial \Omega$;

(iii) $\operatorname{deg}\left(\left.Q N\right|_{\operatorname{Ker} L}, \Omega \cap \operatorname{Ker} L, 0\right) \neq 0$.

Then the equation $L x=N x$ has at least one solution in $\operatorname{dom} L \cap \bar{\Omega}$.

Next, we recall some basic knowledge about the fractional calculus. For more details we refer the reader to [2].

Definition 2.1 The Riemann-Liouville fractional integral of order $\alpha>0$ for a function $x:(0,+\infty) \rightarrow \mathbb{R}$ is given by

$$
I_{0+}^{\alpha} x(t)=\frac{1}{\Gamma(\alpha)} \int_{0}^{t}(t-s)^{\alpha-1} x(s) d s
$$

provided that the right-hand side integral is pointwise defined on $(0,+\infty)$.

Definition 2.2 The Riemann-Liouville fractional derivative of order $\alpha>0$ for a function $x:(0,+\infty) \rightarrow \mathbb{R}$ is given by

$$
D_{0+}^{\alpha} x(t)=\frac{d^{n}}{d t^{n}} I_{0+}^{n-\alpha} x(t)=\frac{1}{\Gamma(n-\alpha)} \frac{d^{n}}{d t^{n}} \int_{0}^{t}(t-s)^{n-\alpha-1} x(s) d s,
$$

where $n=[\alpha]+1$, provided that the right-hand side integral is pointwise defined on $(0,+\infty)$. 
Lemma 2.1 Let $\alpha>0$. Assume that $x, D_{0+}^{\alpha} x \in L^{1}(0,1)$, then

$$
I_{0+}^{\alpha} D_{0+}^{\alpha} x(t)=x(t)+c_{1} t^{\alpha-1}+c_{2} t^{\alpha-2}+\cdots+c_{n} t^{\alpha-n},
$$

where $n=[\alpha]+1, c_{i} \in \mathbb{R}(i=1,2, \ldots, n)$ are arbitrary constants.

Lemma 2.2 Let $\alpha>\beta>0$. Assume that $x \in L^{1}(0,1)$, then

$$
I_{0+}^{\alpha} I_{0+}^{\beta} x(t)=I_{0+}^{\alpha+\beta} x(t), \quad D_{0+}^{\beta} I_{0+}^{\alpha} x(t)=I_{0+}^{\alpha-\beta} x(t),
$$

in particular $D_{0+}^{\alpha} I_{0+}^{\alpha} x(t)=x(t)$.

Lemma 2.3 Let $\alpha>0, n \in N$ and $D=d / d x$. If the fractional derivatives $\left(D_{0_{+}}^{\alpha} x\right)(t)$ and $\left(D_{0+}^{\alpha+n} x\right)(t)$ exist, then

$$
\left(D^{n} D_{0+}^{\alpha} x\right)(t)=\left(D_{0+}^{\alpha+n} x\right)(t)
$$

Lemma 2.4 (see $[2,34])$ Assume that $\alpha>0, \lambda>-1, t>0$, then

$$
I_{0+}^{\alpha} t^{\lambda}=\frac{\Gamma(\lambda+1)}{\Gamma(\lambda+1+\alpha)} t^{\alpha+\lambda}, \quad D_{0+}^{\alpha} t^{\lambda}=\frac{\Gamma(\lambda+1)}{\Gamma(\lambda+1-\alpha)} t^{\lambda-\alpha},
$$

in particular $D_{0+}^{\alpha} t^{\alpha-m}=0, m=1,2, \ldots, n$, where $n=[\alpha]+1$.

\section{Main result}

Let

$$
X=\left\{x: x, D_{0+}^{\alpha-2} x, D_{0+}^{\alpha-1} x \in C[0,1]\right\}, \quad Y=L^{1}[0,1] .
$$

It is easy to check that $X$ is a Banach space with norm

$$
\|x\|_{X}=\max \left\{\|x\|_{\infty},\left\|D_{0+}^{\alpha-2} x\right\|_{\infty},\left\|D_{0+}^{\alpha-1} x\right\|_{\infty}\right\}
$$

where $\|x\|_{\infty}=\sup _{t \in[0,1]}|x(t)|$, and $Y$ is a Banach space with norm $\|y\|_{Y}=\|y\|_{1}=\int_{0}^{1}|y(t)| d t$.

Define the linear operator $L: \operatorname{dom} L \subset X \rightarrow Y$ and the nonlinear operator $N: X \rightarrow Y$ as follows:

$$
L x(t)=D_{0+}^{\alpha} x(t), \quad x(t) \in \operatorname{dom} L, \quad N x(t)=f\left(t, x(t), D_{0+}^{\alpha-2} x(t), D_{0+}^{\alpha-1} x(t)\right), \quad x(t) \in X,
$$

where

$$
\operatorname{dom} L=\left\{x \in X: D_{0+}^{\alpha} x(t) \in Y, x \text { satisfies boundary value conditions of (1.1) }\right\} .
$$

Then BVP (1.1) is equivalent to the operator equation $L x=N x, x \in \operatorname{dom} L$. 
Lemma 3.1 Assume that $\left(\mathrm{H}_{1}\right)$ holds, then the operator $L: \operatorname{dom} L \subset X \rightarrow Y$ satisfies

$$
\begin{aligned}
& \operatorname{Ker} L=\left\{x \in \operatorname{dom} L: x(t)=a_{1} t^{\alpha-1}+a_{2} t^{\alpha-2}, a_{1}, a_{2} \in \mathbb{R}\right\}, \\
& \operatorname{Im} L=\left\{y \in Y: T_{1} y=T_{2} y=0\right\}
\end{aligned}
$$

where

$$
T_{1} y=\sum_{i=1}^{+\infty} \alpha_{i} \int_{0}^{\xi_{i}} y(s) d s, \quad T_{2} y=\sum_{i=1}^{+\infty} \beta_{i} \int_{\gamma_{i}}^{1} y(s) d s .
$$

Proof If $L x=D_{0+}^{\alpha} x=0$, by Lemma 2.1, we have

$$
x(t)=a_{1} t^{\alpha-1}+a_{2} t^{\alpha-2}+a_{3} t^{\alpha-3}, \quad a_{1}, a_{2}, a_{3} \in \mathbb{R} .
$$

Considering that boundary condition $x(0)=0$, one has $a_{3}=0$, then

$$
x(t)=a_{1} t^{\alpha-1}+a_{2} t^{\alpha-2} .
$$

So, $\operatorname{Ker} L \subset\left\{x \in \operatorname{dom} L: x(t)=a_{1} t^{\alpha-1}+a_{2} t^{\alpha-2}, a_{1}, a_{2} \in \mathbb{R}\right\}$. Conversely, for any $a_{1}, a_{2} \in \mathbb{R}$, take $x(t)=a_{1} t^{\alpha-1}+a_{2} t^{\alpha-2}$, it is easy to check that $D_{0+}^{\alpha} x(t)=0$ and $x(t)$ satisfies boundary value conditions of (1.1). Thus, (3.1) holds. For $y \in \operatorname{Im} L$, there exists $x \in \operatorname{dom} L$ such that $D_{0+}^{\alpha} x(t)=y(t)$. Again by Lemma 2.1 and combining with the boundary condition $x(0)=0$, one gets

$$
x(t)=I_{0+}^{\alpha} y(t)+a_{1} t^{\alpha-1}+a_{2} t^{\alpha-2} .
$$

Noting that

$$
D_{0+}^{\alpha-1} x(0)=\sum_{i=1}^{+\infty} \alpha_{i} D_{0+}^{\alpha-1} x\left(\xi_{i}\right), \quad D_{0+}^{\alpha-1} x(1)=\sum_{i=1}^{+\infty} \beta_{i} D_{0+}^{\alpha-1} x\left(\gamma_{i}\right),
$$

by Lemmas 2.2 and 2.4 , we obtain

$$
\begin{aligned}
D_{0+}^{\alpha-1} x(0) & =a_{1} \Gamma(\alpha)=\sum_{i=1}^{+\infty} \alpha_{i} D_{0+}^{\alpha-1} x\left(\xi_{i}\right) \\
& =\sum_{i=1}^{+\infty} \alpha_{i}\left[\int_{0}^{\xi_{i}} y(s) d s+a_{1} \Gamma(\alpha)\right] \\
& =\sum_{i=1}^{+\infty} \alpha_{i} \int_{0}^{\xi_{i}} y(s) d s+a_{1} \Gamma(\alpha)
\end{aligned}
$$

and

$$
\begin{aligned}
D_{0+}^{\alpha-1} x(1) & =\int_{0}^{1} y(s) d s+a_{1} \Gamma(\alpha)=\sum_{i=1}^{+\infty} \beta_{i} D_{0+}^{\alpha-1} x\left(\gamma_{i}\right) \\
& =\sum_{i=1}^{+\infty} \beta_{i}\left[\int_{0}^{\gamma_{i}} y(s) d s+a_{1} \Gamma(\alpha)\right]=\sum_{i=1}^{+\infty} \beta_{i} \int_{0}^{\gamma_{i}} y(s) d s+a_{1} \Gamma(\alpha) .
\end{aligned}
$$


Thus,

$$
T_{1} y=T_{2} y=0
$$

that is,

$$
\operatorname{Im} L \subset\left\{y \in Y: T_{1} y=T_{2} y=0\right\}
$$

Conversely, let $y \in Y$ satisfy (3.3) and take $x(t)=I_{0+}^{\alpha} y(t)$. Obviously, we have $x(t) \in \operatorname{dom} L$ and $L x(t)=y(t)$. Then, $\left\{y \in Y: T_{1} y=T_{2} y=0\right\} \subset \operatorname{Im} L$. Therefore, (3.2) holds.

Let $Q_{1}, Q_{2}: Y \rightarrow Y$ be two linear operators defined as follows:

$$
Q_{1} y=\frac{1}{\Delta}\left(a_{22} T_{1} y-a_{12} T_{2} y\right), \quad Q_{2} y=\frac{2}{\Delta}\left(a_{11} T_{2} y-a_{21} T_{1} y\right) .
$$

Lemma 3.2 Assume that $\left(\mathrm{H}_{1}\right)$ holds, then $L: \operatorname{dom} L \subset X \rightarrow Y$ is a Fredholm operator of index zero. The linear projector operator $P: X \rightarrow X$ and $Q: Y \rightarrow Y$ defined as follows:

$$
\begin{aligned}
& (P x)(t)=\frac{1}{\Gamma(\alpha)} D_{0+}^{\alpha-1} x(0) t^{\alpha-1}+\frac{1}{\Gamma(\alpha-1)} D_{0+}^{\alpha-2} x(0) t^{\alpha-2}, \\
& Q y(t)=Q_{1} y(t)+\left(Q_{2} y(t)\right) t .
\end{aligned}
$$

Proof By the definition of $P$ we can check that $P$ is a continuous linear projector operator and satisfies $\operatorname{Im} P=\operatorname{Ker} L, X=\operatorname{Ker} P \oplus \operatorname{Ker} L$. It is clear that $Q$ is a continuous linear operator and $\operatorname{dim} \operatorname{Im} Q=2$. By the definitions of $Q_{1}, Q_{2}$, we can calculate the following equations hold:

$$
\begin{aligned}
& Q_{1}\left(Q_{1} y(t)\right)=Q_{1} y(t), \quad Q_{1}\left(\left(Q_{2} y(t)\right) t\right)=0, \\
& Q_{2}\left(Q_{1} y(t)\right)=0, \quad Q_{2}\left(\left(Q_{2} y(t)\right) t\right)=Q_{2} y(t) .
\end{aligned}
$$

Thus,

$$
\begin{aligned}
Q^{2} y(t) & =Q(Q y(t))=Q_{1}(Q y(t))+\left(Q_{2}(Q y(t))\right) t \\
& =Q_{1}\left[Q_{1} y(t)+\left(Q_{2} y(t)\right) t\right]+\left\{Q_{2}\left[Q_{1} y(t)+\left(Q_{2} y(t)\right) t\right]\right\} t \\
& =Q_{1} y(t)+\left(Q_{2} y(t)\right) t=Q y(t) .
\end{aligned}
$$

So, $Q$ is a projector operator. From Lemma 3.1, we have $\operatorname{Im} L \subset \operatorname{Ker} Q$. Now, we show the fact that $\operatorname{Ker} Q \subset \operatorname{Im} L$. In fact, for $y \in \operatorname{Ker} Q$, i.e., $Q y=0$, then we get a system of linear equations with respect to $T_{1} y, T_{2} y$ as follows:

$$
\left\{\begin{array}{l}
a_{11} T_{2} y-a_{21} T_{1} y=0 \\
a_{22} T_{1} y-a_{12} T_{2} y=0
\end{array}\right.
$$

Since the determinant of coefficient for (3.4) is $\Delta \neq 0$, we get $T_{1} y=T_{2} y=0$, thus $\operatorname{Ker} Q \subset$ $\operatorname{Im} L$. Therefore, $\operatorname{Ker} Q=\operatorname{Im} L$. For $y \in Y$, set $y=(y-Q y)+Q y$, then $(y-Q y) \in \operatorname{Ker} Q=\operatorname{Im} L$, 
$Q y \in \operatorname{Im} Q$. So, $y=\operatorname{Im} L+\operatorname{Im} Q$. Furthermore, for any $y \in \operatorname{Im} L \cap \operatorname{Im} Q$, there exist constants $c_{1}, c_{2} \in \mathbb{R}$ such that $y(t)=c_{1}+c_{2} t$ and $T_{1} y=T_{2} y=0$. Then we also get a system of linear equations with respect to $c_{1}, c_{2}$ as follows:

$$
\left\{\begin{array}{l}
2 a_{11} c_{1}+a_{12} c_{2}=0, \\
2 a_{21} c_{1}+a_{22} c_{2}=0 .
\end{array}\right.
$$

Because the determinant of coefficient for (3.5) is $2 \Delta \neq 0$. Thus, $c_{1}=c_{2}=0$. It means that $\operatorname{Im} Q \cap \operatorname{Im} L=\{0\}$. Therefore, $Y=\operatorname{Im} Q \oplus \operatorname{Im} L$. Furthermore, $\operatorname{dimKer} L=\operatorname{dim} \operatorname{Im} Q=$ $\operatorname{codim} \operatorname{Im} L=2$. So, $L$ is a Fredholm operator of index zero.

Lemma 3.3 Assume that $\left(\mathrm{H}_{1}\right)$ holds, define the linear operator $K_{p}: \operatorname{Im} L \rightarrow \operatorname{dom} L \cap \operatorname{Ker} P$ by

$$
\left(K_{p} y\right)(t)=\frac{1}{\Gamma(\alpha)} \int_{0}^{t}(t-s)^{\alpha-1} y(s) d s, \quad y \in \operatorname{Im} L,
$$

then $K_{p}$ is the inverse of $\left.L\right|_{\operatorname{dom} L \cap \operatorname{Ker} P}$ and $\left\|K_{p} y\right\|_{X} \leq\|y\|_{1}$, for all $y \in \operatorname{Im} L$.

Proof For $y \in \operatorname{Im} L$, then $T_{1} y=T_{2} y=0$, which is combined with the definition of $K_{p}$ and Lemma 2.2, we can check that $K_{p} y \in \operatorname{dom} L \cap \operatorname{Ker} P$. So, $K_{p}$ is well defined on $\operatorname{Im} L$. Obviously, $\left(L K_{p}\right) y(t)=y(t), \forall y \in \operatorname{Im} L$. For $x(t) \in \operatorname{dom} L$, by Lemma 2.1, we have

$$
\begin{aligned}
\left(K_{p} L\right) x(t) & =I_{0+}^{\alpha} D_{0+}^{\alpha} x(t) \\
& =x(t)+a_{1} t^{\alpha-1}+a_{2} t^{\alpha-2}, \quad a_{1}, a_{2} \in \mathbb{R} .
\end{aligned}
$$

It follows from $P\left[\left(K_{p} L\right) x(t)\right]=0$ and $a_{1} t^{\alpha-1}+a_{2} t^{\alpha-2} \in \operatorname{Ker} L=\operatorname{Im} P$ that $c_{1} t^{\alpha-1}+c_{2} t^{\alpha-2}=$ $-P x(t)$. That is, $\left(K_{p} L\right) x(t)=x(t)-P x(t)$. Therefore, if $x(t) \in \operatorname{dom} L \cap \operatorname{Ker} P$, we have $\left(K_{p} L\right) x(t)=x(t)$. So, $K_{p}$ is the inverse of $\left.L\right|_{\operatorname{dom} L \cap K e r P}$. By Lemma 2.2, we have the following inequalities:

$$
\begin{aligned}
& \left|K_{p} y\right| \leq \frac{1}{\Gamma(\alpha)} \int_{0}^{t}(t-s)^{\alpha-1}|y(s)| d s \leq \frac{1}{\Gamma(\alpha)} \int_{0}^{1}|y(s)| d s \leq\|y\|_{1}, \\
& \left|D_{0+}^{\alpha-2} K_{p} y\right| \leq \int_{0}^{t}(t-s)|y(s)| d s \leq \int_{0}^{1}|y(s)| d s=\|y\|_{1}, \\
& \left|D_{0+}^{\alpha-1} K_{p} y\right| \leq \int_{0}^{t}|y(s)| d s \leq \int_{0}^{1}|y(s)| d s=\|y\|_{1} .
\end{aligned}
$$

So, $\left\|K_{p} y\right\|_{X} \leq\|y\|_{1}$, for all $y \in \operatorname{Im} L$.

Lemma 3.4 Assume that $\left(\mathrm{H}_{1}\right)$ holds and $\Omega \subset X$ is an open bounded subset with $\operatorname{dom} L \cap$ $\bar{\Omega} \neq \emptyset$, then $N$ is L-compact on $\bar{\Omega}$.

Proof According to $f \in[0,1] \times \mathbb{R}^{2} \rightarrow \mathbb{R}$ satisfies the Carathéodory conditions, we can get $Q N(\bar{\Omega})$ and $(I-Q) N(\bar{\Omega})$ are bounded almost everywhere on $[0,1]$, that is, there exist constants $m, \tilde{m}>0$ such that $|Q N x(t)| \leq \tilde{m},|(I-Q) N x(t)| \leq m, x \in \bar{\Omega}$, a.e. $t \in[0,1]$. Next, we show that $K_{p}(I-Q) N: \bar{\Omega} \rightarrow X$ is compact. In fact, by Lemma 3.3, $K_{p}(I-Q) N(\bar{\Omega})$ is 
uniformly bounded. It follows from the Lebesgue dominated convergence theorem that $K_{p}(I-Q) N: \bar{\Omega} \rightarrow X$ is continuous. For $0 \leq t_{1}<t_{2} \leq 1, x \in \bar{\Omega}$, we have

$$
\begin{aligned}
\left|K_{p}(I-Q) N x\left(t_{1}\right)-K_{p}(I-Q) N x\left(t_{2}\right)\right| \\
=\frac{1}{\Gamma(\alpha)}\left|\int_{0}^{t_{1}}\left(t_{1}-s\right)^{\alpha-1}(I-Q) N x(s) d s-\int_{0}^{t_{2}}\left(t_{2}-s\right)^{\alpha-1}(I-Q) N x(s) d s\right| \\
\leq \frac{1}{\Gamma(\alpha)}\left|\int_{0}^{t_{1}}\left[\left(t_{1}-s\right)^{\alpha-1}-\left(t_{2}-s\right)^{\alpha-1}\right](I-Q) N x(s) d s\right| \\
\quad+\frac{1}{\Gamma(\alpha)}\left|\int_{t_{1}}^{t_{2}}\left(t_{2}-s\right)^{\alpha-1}(I-Q) N x(s) d s\right| \\
\leq \frac{m}{\Gamma(\alpha)} \int_{0}^{t_{1}}\left[\left(t_{2}-s\right)^{\alpha-1}-\left(t_{1}-s\right)^{\alpha-1}\right] d s+\frac{m}{\Gamma(\alpha)} \int_{t_{1}}^{t_{2}}\left(t_{2}-s\right)^{\alpha-1} d s \\
=\frac{m}{\Gamma(\alpha+1)}\left(t_{2}^{\alpha}-t_{1}^{\alpha}\right) .
\end{aligned}
$$

Since $t^{\alpha}$ is uniformly continuous on $[0,1], K_{p}(I-Q) N(\bar{\Omega})$ is equicontinuous. In addition, by Lemma 2.3 we see that the following equation holds:

$$
D_{0+}^{\alpha-2} K_{p}(I-Q) N x\left(t_{2}\right)-D_{0+}^{\alpha-2} K_{p}(I-Q) N x\left(t_{1}\right)=\int_{t_{1}}^{t_{2}} D_{0+}^{\alpha-1} K_{p}(I-Q) N x(s) d s .
$$

So, it suffices to show that $D_{0+}^{\alpha-1} K_{p}(I-Q) N(\bar{\Omega})$ is equicontinuous. In fact,

$$
\begin{aligned}
& \left|D_{0+}^{\alpha-1} K_{p}(I-Q) N x\left(t_{1}\right)-D_{0+}^{\alpha-1} K_{p}(I-Q) N x\left(t_{2}\right)\right| \\
& \quad=\left|\int_{t_{1}}^{t_{2}}(I-Q) N x(s) d s\right| \leq m\left(t_{2}-t_{1}\right) .
\end{aligned}
$$

Since $t$ is uniformly continuous on $[0,1]$, thus $D_{0+}^{\alpha-1} K_{p}(I-Q) N x(\Omega)$ is equicontinuous. By the Ascoli-Arzelà theorem, we see that $K_{p}(I-Q) N: \bar{\Omega} \rightarrow X$ is compact.

In order to obtain our main results, we suppose that the following conditions are satisfied:

$\left(\mathrm{H}_{2}\right)$ There exist nonnegative functions $p(t), q(t), r(t), e(t) \in Y$ such that, for all $(u, v, w) \in$ $\mathbb{R}^{3}, t \in(0,1)$,

$$
|f(t, u, v, w)| \leq p(t)|u|+q(t)|v|+r(t)|w|+e(t)
$$

and

$$
\|p\|_{1}+\|q\|_{1}+\|r\|_{1}<\frac{\Gamma(\alpha-1)}{\Gamma(\alpha-1)+2} .
$$

$\left(\mathrm{H}_{3}\right)$ There exist constants $k_{1}, k_{2}>0$ such that, for all $t \in(0,1), x \in \operatorname{dom} L$, if $\left|D_{0_{+}}^{\alpha-2} x(t)\right|>$ $k_{1}$ or $\left|D_{0+}^{\alpha-1} x(t)\right|>k_{2}$, then either $T_{1}(N x(t)) \neq 0$ or $T_{2}(N x(t)) \neq 0$.

$\left(\mathrm{H}_{4}\right)$ There exists a constant $g>0$ such that, for all $a_{1}, a_{2} \in \mathbb{R}, x(t)=a_{1} t^{\alpha-1}+a_{2} t^{\alpha-2} \in$ $\operatorname{Ker} L$, if $\left|a_{1}\right|>g$ or $\left|a_{2}\right|>g$ then either

$$
a_{1} T_{1} N x(t)+a_{2} T_{2} N x(t)>0
$$


or

$$
a_{1} T_{1} N x(t)+a_{2} T_{2} N x(t)<0 .
$$

Lemma 3.5 Suppose that $\left(\mathrm{H}_{1}\right)-\left(\mathrm{H}_{3}\right)$ hold, set

$$
\Omega_{1}=\{x \in \operatorname{dom} L \backslash \operatorname{Ker} L: L x=\lambda N x, \lambda \in(0,1)\} .
$$

Then $\Omega_{1}$ is bounded.

Proof For $x \in \Omega_{1}$, we have $N x \in \operatorname{Im} L=\operatorname{Ker} Q$. Then $T_{1}(N x(t))=T_{2}(N x(t))=0$. Thus, from $\left(\mathrm{H}_{3}\right)$, there exist $t_{0}, t_{1} \in[0,1]$ such that $\left|D_{0+}^{\alpha-2} x\left(t_{0}\right)\right| \leq k_{1}$ and $\left|D_{0+}^{\alpha-1} x\left(t_{1}\right)\right| \leq k_{2}$. Then, by Lemma 2.3 we have

$$
\begin{aligned}
\left|D_{0+}^{\alpha-1} x(t)\right| & =\left|D_{0+}^{\alpha-1} x\left(t_{1}\right)+\int_{t_{1}}^{t} D_{0+}^{\alpha} x(s) d s\right| \\
& \leq\left|D_{0+}^{\alpha-1} x\left(t_{1}\right)\right|+\int_{t_{1}}^{t}\left|D_{0+}^{\alpha} x(s)\right| d s \\
& \leq k_{2}+\|N x\|_{1}
\end{aligned}
$$

and

$$
\begin{aligned}
\left|D_{0+}^{\alpha-2} x(0)\right| & =\left|D_{0+}^{\alpha-2} x\left(t_{0}\right)-\int_{0}^{t_{0}} D_{0+}^{\alpha-1} x(s) d s\right| \\
& \leq\left|D_{0+}^{\alpha-2} x\left(t_{0}\right)\right|+\int_{0}^{t_{0}}\left|D_{0+}^{\alpha-1} x(s)\right| d s \\
& \leq k_{1}+\left\|D_{0+}^{\alpha-1} x\right\|_{\infty} \leq k_{1}+k_{2}+\|N x\|_{1} .
\end{aligned}
$$

By the definition of $P$ and Lemma 2.4 , we get

$$
D_{0+}^{\alpha-2} P x(t)=D_{0+}^{\alpha-1} x(0) t+D_{0+}^{\alpha-2} x(0)
$$

and

$$
D_{0+}^{\alpha-1} P x(t)=D_{0+}^{\alpha-1} x(0) .
$$

Thus,

$$
\begin{aligned}
& \left|D_{0+}^{\alpha-2} P x\right| \leq\left|D_{0+}^{\alpha-1} x(0)\right|+\left|D_{0+}^{\alpha-2} x(0)\right| \leq k_{1}+2 k_{2}+2\|N x\|_{1}, \\
& \left|D_{0+}^{\alpha-1} P x\right|=\left|D_{0+}^{\alpha-1} x(0)\right| \leq k_{2}+\|N x\|_{1} .
\end{aligned}
$$

We have

$$
\begin{aligned}
|P x| & \leq \frac{1}{\Gamma(\alpha)}\left|D_{0+}^{\alpha-1} x(0)\right|+\frac{1}{\Gamma(\alpha-1)}\left|D_{0+}^{\alpha-2} x(0)\right| \\
& \leq \frac{1}{\Gamma(\alpha-1)}\left(k_{1}+2 k_{2}+2\|N x\|_{1}\right) .
\end{aligned}
$$


Therefore,

$$
\begin{aligned}
\|P x\|_{X} & =\max \left\{\|P x\|_{\infty},\left\|D_{0+}^{\alpha-2} P x\right\|_{\infty},\left\|D_{0+}^{\alpha-1} P x\right\|_{\infty}\right\} \\
& \leq \frac{1}{\Gamma(\alpha-1)}\left(k_{1}+2 k_{2}+2\|N x\|_{1}\right) .
\end{aligned}
$$

Also, for $x \in \Omega_{1}$, then $(I-P) x \in \operatorname{dom} L \cap \operatorname{Ker} P, L P x=0$, from Lemma 3.3, we have

$$
\|(I-P) x\|_{X}=\left\|K_{p} L(I-P) x\right\|_{X}=\left\|K_{p} L x\right\|_{X} \leq\|L x\|_{1} \leq\|N x\|_{1} .
$$

Then, from (3.8) and (3.9), we see that

$$
\begin{aligned}
\|x\|_{X} & =\|P x+(I-P) x\|_{X} \leq\|P x\|_{X}+\|(I-P) x\|_{X} \\
& \leq \frac{1}{\Gamma(\alpha-1)}\left(k_{1}+2 k_{2}+2\|N x\|_{1}\right)+\|N x\|_{1} .
\end{aligned}
$$

By $\left(\mathrm{H}_{2}\right)$, we have

$$
\begin{aligned}
\|N x\|_{1} & \leq\|p\|_{1}\|x\|_{\infty}+\|q\|_{1}\left\|D_{0_{+}}^{\alpha-2} x\right\|_{\infty}+\|r\|_{1}\left\|D_{0+}^{\alpha-1} x\right\|_{\infty}+\|e\|_{1} \\
& \leq\left(\|p\|_{1}+\|q\|_{1}+\|r\|_{1}\right)\|x\|_{X}+\|e\|_{1} .
\end{aligned}
$$

Substituting (3.11) into (3.10), one gets

$$
\|x\|_{X} \leq \frac{k_{1}+2 k_{2}+(\Gamma(\alpha-1)+2)\|e\|_{1}}{\Gamma(\alpha-1)-[\Gamma(\alpha-1)+2]\left(\|p\|_{1}+\|q\|_{1}+\|r\|_{1}\right)} .
$$

So, $\Omega_{1}$ is bounded.

Lemma 3.6 Suppose that $\left(\mathrm{H}_{3}\right)$ holds, set

$$
\Omega_{2}=\{x \in \operatorname{Ker} L: N x \in \operatorname{Im} L\}
$$

Then $\Omega_{2}$ is bounded.

Proof For $x \in \Omega_{2}$, we have $x(t)=a_{1} t^{\alpha-1}+a_{2} t^{\alpha-2}, a_{1}, a_{2} \in \mathbb{R}$ and $T_{1} N x(t)=T_{2} N x(t)=0$. From $\left(\mathrm{H}_{3}\right)$, there exist $t_{2}, t_{3} \in[0,1]$ such that $\left|D_{0+}^{\alpha-2} x\left(t_{2}\right)\right| \leq k_{1}$ and $\left|D_{0+}^{\alpha-1} x\left(t_{3}\right)\right| \leq k_{2}$, that is,

$$
\begin{aligned}
& \left|D_{0+}^{\alpha-1} x\left(t_{3}\right)\right|=\left|a_{1} \Gamma(\alpha)\right| \leq k_{2}, \\
& \left|D_{0+}^{\alpha-2} x\left(t_{2}\right)\right|=\left|a_{1} \Gamma(\alpha) t_{2}+a_{2} \Gamma(\alpha-1)\right| \leq k_{1} .
\end{aligned}
$$

Thus,

$$
\left|a_{1}\right| \leq k_{2} / \Gamma(\alpha), \quad\left|a_{2}\right| \leq\left(k_{1}+k_{2}\right) / \Gamma(\alpha-1) .
$$


Therefore,

$$
\begin{aligned}
& \|x\|_{\infty} \leq\left|a_{1}\right|+\left|a_{2}\right| \leq \frac{k_{2}}{\Gamma(\alpha)}+\frac{k_{1}+k_{2}}{\Gamma(\alpha-1)}=\frac{1}{\Gamma(\alpha)}\left[k_{2}+(\alpha-1)\left(k_{1}+k_{2}\right)\right], \\
& \left\|D_{0+}^{\alpha-2} x\right\|_{\infty} \leq \Gamma(\alpha)\left|a_{1}\right|+\Gamma(\alpha-1)\left|a_{2}\right| \leq 2 k_{2}+k_{1}, \\
& \left\|D_{0+}^{\alpha-1} x\right\|_{\infty} \leq \Gamma(\alpha)|a| \leq k_{2} .
\end{aligned}
$$

So, $\Omega_{2}$ is bounded.

Lemma 3.7 Suppose that $\left(\mathrm{H}_{4}\right)$ holds, we set

$$
\Omega_{3}=\{x \in \operatorname{Ker} L: \vartheta \lambda J x+(1-\lambda) Q N x=0, \lambda \in[0,1]\} .
$$

Then $\Omega_{3}$ is bounded, where $\vartheta=1$, if (3.6) holds and $\vartheta=-1$, if (3.7) holds, $J: \operatorname{Ker} L \rightarrow \operatorname{Im} Q$ is the linear isomorphism defined by

$$
J\left(a_{1} t^{\alpha-1}+a_{2} t^{\alpha-2}\right)=\frac{1}{\Delta}\left[\left(a_{22} a_{1}-a_{12} a_{2}\right)+2\left(a_{11} a_{2}-a_{21} a_{1}\right) t\right], \quad \forall a_{1}, a_{2} \in \mathbb{R} .
$$

Proof Without loss of generality, we suppose that (3.7) holds, then, for any $x \in \Omega_{3}$, there exist constants $a_{1}, a_{2} \in \mathbb{R}, \lambda \in[0,1]$ such that $x(t)=a_{1} t^{\alpha-1}+a_{2} t^{\alpha-2}$ and $-\lambda J x+$ $(1-\lambda) Q N x=0$. By Lemma 3.6, in order to prove Lemma 3.7, it suffices to show that $\left|a_{1}\right| \leq g,\left|a_{2}\right| \leq g$. In fact, if $\lambda=0$ then $Q N x=0$, which means $T_{1} N x=T_{2} N x=0$. From $\left(\mathrm{H}_{4}\right)$, we get $\left|a_{1}\right| \leq g,\left|a_{2}\right| \leq g$. If $\lambda=1$ then $J x=0$, that is, $a_{1}=a_{2}=0$. Obviously, $\left|a_{1}\right| \leq g$, $\left|a_{2}\right| \leq g$. For $\lambda \in(0,1)$, by $\lambda J x=(1-\lambda) Q N x$ one has

$$
\left\{\begin{array}{l}
\lambda\left(a_{22} a_{1}-a_{12} a_{2}\right)=(1-\lambda)\left(a_{22} T_{1} N x-a_{12} T_{2} N x\right), \\
\lambda\left(a_{11} a_{2}-a_{21} a_{1}\right)=(1-\lambda)\left(a_{11} T_{2} N x-a_{21} T_{1} N x\right) .
\end{array}\right.
$$

Because $\Delta \neq 0$, we have

$$
\left\{\begin{array}{l}
\lambda a_{1}=(1-\lambda) T_{1} N x \\
\lambda a_{2}=(1-\lambda) T_{2} N x
\end{array}\right.
$$

Then, if $\left|a_{1}\right|>g$ or $\left|a_{2}\right|>g$, by (3.7), we get a contradiction,

$$
0<\lambda\left(a_{1}^{2}+a_{2}^{2}\right)=(1-\lambda)\left(a_{1} T_{1} N x+a_{2} T_{2} N x\right)<0 .
$$

Thus, $\left|a_{1}\right| \leq g,\left|a_{2}\right| \leq g$. So, $\Omega_{3}$ is bounded. If (3.6) holds, by a similar method, we can see that $\Omega_{3}$ is bounded.

Theorem 3.1 Suppose that $\left(\mathrm{H}_{1}\right)-\left(\mathrm{H}_{4}\right)$ hold. Then problem (1.1) has at least on solution in $X$.

Proof Let $\Omega$ be a bounded open set of $X$ such that $\bigcup_{i=1}^{3} \bar{\Omega}_{i} \subset \Omega$. By Lemma 3.4, $N$ is $L$ compact on $\bar{\Omega}$. From Lemmas 3.5 and 3.6, we get 
(i) $L x \neq \lambda N x$ for any $(x, \lambda) \in[(\operatorname{dom} L \backslash \operatorname{Ker} L) \cap \partial \Omega] \times(0,1)$,

(ii) $N x \in \operatorname{Im} L$ for any $x \in \operatorname{Ker} L \cap \partial \Omega$.

Thus, we only need to show that (iii) of Theorem 2.1 is satisfied. Take

$$
H(x, \lambda)=\vartheta \lambda J x+(1-\lambda) Q N x,
$$

where $\vartheta$ is defined as before. According to Lemma 3.7, we derive $H(x, \lambda) \neq 0$ for all $x \in$ $\operatorname{Ker} L \cap \partial \Omega$. Thus, it follows from the homotopy of degree that

$$
\begin{aligned}
\operatorname{deg}\left\{\left.Q N\right|_{\text {Ker } L}, \Omega \cap \operatorname{Ker} L, 0\right\} & =\operatorname{deg}\{H(\cdot, 0), \Omega \cap \operatorname{Ker} L, 0\} \\
& =\operatorname{deg}\{H(\cdot, 1), \Omega \cap \operatorname{Ker} L, 0\} \\
& =\operatorname{deg}(\vartheta J, \Omega \cap \operatorname{Ker} L, 0) \neq 0 .
\end{aligned}
$$

Then, by Theorem 2.1, we can see that the operator function $L x=N x$ has at least one solution in $\operatorname{dom} L \cap \bar{\Omega}$, which, equivalently to problem (1.1), has at least one solution in $X$.

\section{Example}

Example 4.1 Consider the following fractional boundary value problem:

$$
\left\{\begin{array}{l}
D_{0+}^{2.5} x(t)=f\left(t, x(t), D_{0+}^{0.5} x(t), D_{0+}^{1.5} x(t)\right), \quad t \in(0,1) \\
x(0)=0, \quad D_{0+}^{1.5} x(0)=\sum_{i=1}^{+\infty} \alpha_{i} D_{0+}^{1.5} x\left(\xi_{i}\right) \\
D_{0+}^{1.5} x(1)=\sum_{i=1}^{+\infty} \beta_{i} D_{0+}^{1.5} x\left(\gamma_{i}\right)
\end{array}\right.
$$

where we take

$$
\begin{aligned}
& \alpha_{i}=\frac{1}{2^{i}}, \quad \beta_{i}=\frac{1}{2^{i}}, \quad \xi_{i}=\frac{i}{2 i+1}, \quad \gamma_{i}=\frac{i+1}{2 i+1}, \\
& f\left(t, x(t), D_{0+}^{0.5} x(t), D_{0+}^{1.5} x(t)\right) \\
& \quad=m(t)\left[t^{2}+1+\frac{1}{8} \cos ^{2} x(t)+\frac{1}{24} D_{0+}^{0.5} x(t)\right]-\frac{1}{12}(1-m(t)) D_{0+}^{1.5} x(t), \\
& m(t)= \begin{cases}1, & {[0,1 / 2),} \\
0, & {[1 / 2,1] .}\end{cases}
\end{aligned}
$$

Obviously, we have

$$
\alpha_{i}, \beta_{i} \in \mathbb{R}^{+}, \quad a^{2}=\frac{1}{4} \leq \frac{1}{3}=\xi_{1}, \quad \gamma_{1}^{2}=\frac{4}{9} \leq \frac{1}{2}=b,
$$

and $\left\{\xi_{i}\right\}_{i=1}^{+\infty}$ is a monotone increasing sequence and $\left\{\gamma_{i}\right\}_{i=1}^{+\infty}$ is a monotone decreasing sequence. Applying Remark 1.1 we conclude that $\Delta>0$. Let

$$
p(t)=1 / 8, \quad q(t)=1 / 24, \quad r(t)=1 / 12, \quad e(t)=2 .
$$

Then

$$
|f(t, u, v, w)| \leq p(t)|u|+q(t)|v|+r(t)|w|+e(t)
$$


and

$$
\|p\|_{1}+\|q\|_{1}+\|r\|_{1}=\frac{1}{4}<\frac{\Gamma(\alpha-1)}{\Gamma(\alpha-1)+2}=\frac{\sqrt{\pi}}{\sqrt{\pi}+4} .
$$

Take $k_{1}=48, k_{2}=24$. Then, if $\left|D_{0_{+}}^{0.5} x(t)\right|>k_{1}$, one has

$$
T_{1}(N x(t))=\sum_{i=1}^{+\infty} \alpha_{i} \int_{0}^{\xi_{i}} f\left(s, x(s), D_{0+}^{0.5} x(s), D_{0+}^{1.5} x(s)\right) d s \neq 0,
$$

and if $\left|D_{0+}^{1.5} x(t)\right|>k_{2}$, one gets

$$
T_{2}(N x(t))=\sum_{i=1}^{+\infty} \beta_{i} \int_{\gamma_{i}}^{1} f\left(s, x(s), D_{0+}^{0.5} x(s), D_{0+}^{1.5} x(s)\right) d s \neq 0 .
$$

Let $g=261$. Then if $\left|a_{1}\right|>g$, we have

$$
\begin{aligned}
& a_{1} T_{1} N x(t)+a_{2} T_{2} N x(t) \\
&=a_{1} \sum_{i=1}^{+\infty} \alpha_{i} \int_{0}^{\xi_{i}} f\left(s, x(s), D_{0+}^{0.5} x(s), D_{0+}^{1.5} x(s)\right) d s \\
&+a_{2} \sum_{i=1}^{+\infty} \beta_{i} \int_{\gamma_{i}}^{1} f\left(s, x(s), D_{0+}^{0.5} x(s), D_{0+}^{1.5} x(s)\right) d s \\
&=a_{1} \sum_{i=1}^{+\infty} \alpha_{i} \int_{0}^{\xi_{i}}\left[s^{2}+1+\frac{1}{8} \cos ^{2} x(s)+\frac{1}{24}\left(a_{1} \Gamma(1.5) s+a_{2} \Gamma(0.5)\right)\right] d s \\
& \quad-\frac{a_{2}}{12} \sum_{i=1}^{+\infty} \beta_{i} \int_{\gamma_{i}}^{1} a_{1} \Gamma(1.5) d s \\
&=a_{1} \sum_{i=1}^{+\infty} \alpha_{i} \int_{0}^{\xi_{i}}\left(s^{2}+1+\frac{1}{8} \cos ^{2} x(s)\right) d s+\frac{1}{48} \Gamma(1.5) a_{1}^{2} \sum_{i=1}^{+\infty} \alpha_{i} \xi_{i}^{2} \\
&+\frac{1}{24} a_{1} a_{2} \Gamma(0.5) \sum_{i=1}^{+\infty} \alpha_{i} \xi_{i}-\frac{1}{12} a_{1} a_{2} \Gamma(1.5) \sum_{i=1}^{+\infty} \beta_{i}\left(1-\gamma_{i}\right) \\
&= a_{1} \sum_{i=1}^{+\infty} \alpha_{i} \int_{0}^{\xi_{i}}\left(s^{2}+1+\frac{1}{8} \cos ^{2} x(s)\right) d s+\frac{1}{48} \Gamma(1.5) a_{1}^{2} \sum_{i=1}^{+\infty} \alpha_{i} \xi_{i}^{2} \\
& \geq \frac{1}{864} a_{1}^{2}-\frac{29}{48} a_{1}>0 .
\end{aligned}
$$

In view of Theorem 3.1, boundary value problem (4.1) has at least one solution.

Remark 4.1 By the example, we find that it is more difficult to give an infinite series $\sum_{i=1}^{+\infty} \alpha_{i}$ $\left(\sum_{i=1}^{+\infty} \alpha_{i}=1, \sum_{i=1}^{+\infty}\left|\alpha_{i}\right|<+\infty\right)$ than to give a function $g(t) \in L^{1}[0,1]\left(\int_{0}^{1} g(t) d t=1\right)$. For example, for $\int_{0}^{1} g(t)=1$, we can take $g(t) \equiv 1$.

\section{Conclusion}

In this paper, we are focused on investigating the existence of solutions for a class of FDEs with infinite point BCs. By using Mawhin's continuation theorem, an existence theorem 
is established and we also give an example to illustrate the application of the theorem. To the best of our knowledge, the issue on the existence of solutions for infinite point BVPs was first studied by Ma (see [35]) and discussed widely in recent years. It is an interesting question and there is some work to be done in the future, such as: discussing the existence of solutions for $p$-Laplacian FDEs with infinite point BCs at resonance in the case $\operatorname{dim} \operatorname{Ker} L=2$ for one fractional differential equation.

\section{Funding}

This research is supported by the National Natural Science Foundation of China (11271364).

\section{Competing interests}

The authors declare that they have no competing interests.

\section{Authors' contributions}

The authors contributed equally in this article. They have all read and approved the final manuscript.

\section{Publisher's Note}

Springer Nature remains neutral with regard to jurisdictional claims in published maps and institutional affiliations.

Received: 27 December 2017 Accepted: 11 March 2018 Published online: 20 March 2018

\section{References}

1. Podlubny, I.: Fractional Differential Equations: An Introduction to Fractional Derivatives, Fractional Differential Equations, to Methods of Their Solution and Some of Their Applications. Mathematics in Science and Engineering. Academic Press, New York (1999)

2. Kilbas, A.A., Srivastava, H.M., Trujillo, J.J.: Theory and Applications of Fractional Differential Equations. North-Holland Mathematics Studies. Elsevier, Amsterdam (2006)

3. Petráś, I.: Fractional-Order Nonlinear Systems: Modeling, Analysis and Simulation. Springer, Berlin (2011)

4. Sierociuk, D., Dzieliński, A., Sarwas, G., Podlubny, I., Skovranek, T.: Modelling heat transfer in heterogeneous media using fractional calculus. Philos. Trans. R. Soc., Math. Phys. Eng. Sci. 371, 20120146 (2013)

5. Arenas, A.J., González-Parra, G., Chen-Charpentier, B.M.: Construction of nonstandard finite difference schemes for the SI and SIR epidemic models of fractional order. Math. Comput. Simul. 121, 48-63 (2016)

6. Teka, W.W., Upadhyay, P.K., Mondal, A.: Spiking and bursting patterns of fractional-order Izhikevich model. Commun. Nonlinear Sci. Numer. Simul. 56, 161-176 (2018)

7. Ates, I., Zegeling, P.A.: A homotopy perturbation method for fractional-order advection-diffusion-reaction boundary-value problems. Appl. Math. Model. 47, 425-441 (2017)

8. Ahmad, B., Ntouyas, S.K., Alsaedi, A.: On a coupled system of fractional differential equations with coupled nonlocal and integral boundary conditions. Chaos Solitons Fractals 83, 234-241 (2016)

9. Agarwal, R.P., Ahmad, B., Garout, D., Alsaedi, A.: Existence results for coupled nonlinear fractional differential equations equipped with nonlocal coupled flux and multi-point boundary conditions. Chaos Solitons Fractals 102, 149-161 (2017)

10. Günendi, M., Yaslan, I.: Positive solutions of higher-order nonlinear multi-point fractional equations with integral boundary conditions. Fract. Calc. Appl. Anal. 19, 989-1009 (2016)

11. Zhang, X.Q.., Zhong, Q.Y.: Triple positive solutions for nonlocal fractional differential equations with singularities both on time and space variables. Appl. Math. Lett. 80, 12-19 (2018)

12. Zhang, X.Q.., Zhong, Q.Y.: Uniqueness of solution for higher-order fractional differential equations with conjugate type integral conditions. Fract. Calc. Appl. Anal. 20, 1471-1484 (2017)

13. Zhang, W., Liu, W.B.: Existence of solutions for fractional multi-point boundary value problems at resonance with three-dimensional kernels. Adv. Differ. Equ. 2018, 15 (2018)

14. Zhang, W., Liu, W.B., Chen, T.Y.: Solvability for a fractional $p$-Laplacian multipoint boundary value problem at resonance on infinite interval. Adv. Differ. Equ. 2016, 183 (2016)

15. Shen, T.F., Liu, W.B., Shen, X.H.: Existence and uniqueness of solutions for several BVPs of fractional differential equations with $p$-Laplacian operator. Mediterr. J. Math. 13, 4623-4637 (2016)

16. Kosmatov, N., Jiang, W.H.: Resonant functional problems of fractional order. Chaos Solitons Fractals 91, 573-579 (2016)

17. Iqbal, M., Li, Y.J., Shah, K., Khan, R.A.: Application of topological degree method for solutions of coupled systems of multipoints boundary value problems of fractional order hybrid differential equations. Complexity 2017, Article ID 7676814 (2017)

18. Zhang, X.Q.: Positive solutions for a class of singular fractional differential equation with infinite-point boundary value conditions. Appl. Math. Lett. 39, 22-27 (2015)

19. Zhai, C.B., Wang, L.: Some existence, uniqueness results on positive solutions for a fractional differential equation with infinite-point boundary conditions. Nonlinear Anal., Model. Control 22, 566-577 (2017)

20. Guo, L.M., Liu, L.S., Wu, Y.H.: Existence of positive solutions for singular fractional differential equations with infinite-point boundary conditions. Nonlinear Anal., Model. Control 21, 635-650 (2016)

21. Ge, F.D., Zhou, H.C., Kou, C.H.: Existence of solutions for a coupled fractional differential equations with infinitely many points boundary conditions at resonance on an unbounded domain. Differ. Equ. Dyn. Syst. (2016). https://doi.org/10.1007/s12591-015-0270-x 
22. Hu, L., Zhang, S.Q.: Existence results for a coupled system of fractional differential equations with $p$-Laplacian operator and infinite-point boundary conditions. Bound. Value Probl. 2017, 88 (2017)

23. $\mathrm{Hu}, \mathrm{L}$ : Existence of solutions to a coupled system of fractional differential equations with infinite-point boundary value conditions at resonance. Adv. Differ. Equ. 2016, 200 (2016)

24. Guo, L.M., Liu, L.S., Wu, Y.H.: Existence of positive solutions for singular higher-order fractional differential equations with infinite-point boundary conditions. Bound. Value Probl. 2016, 114 (2016)

25. Liu, S.L., Liu, J.P., Dai, Q., Li, H.L.: Uniqueness results for nonlinear fractional differential equations with infinite-point integral boundary conditions. J. Nonlinear Sci. Appl. 10, 1281-1288 (2017)

26. Leng, Q.Q., Yin, J.D., Yan, P.H.: Existence and uniqueness of positive solutions for singular higher order fractional differential equations with infinite-point boundary value conditions. J. Comput. Anal. Appl. 25, 302-310 (2018)

27. Li, X.C., Liu, X.P., Jia, M., Zhang, L.C.: The positive solutions of infinite-point boundary value problem of fractional differential equations on the infinite interval. Adv. Differ. Equ. 2017, 126 (2017)

28. Wang, L., Zhai, C.B.: Uniqueness and properties of positive solutions for infinite-point fractional differential equation with p-Laplacian and a parameter. J. Nonlinear Sci. Appl. 10, 5156-5164 (2017)

29. Qiao, Y., Zhou, Z.F.: Existence of positive solutions of singular fractional differential equations with infinite-point boundary conditions. Adv. Differ. Equ. 2017, 8 (2017)

30. Zhong, Q.Y., Zhang, X.Q.: Positive solution for higher-order singular infinite-point fractional differential equation with p-Laplacian. Adv. Differ. Equ. 2016, 11 (2016)

31. Li, B.X., Sun, S.R., Sun, Y.: Existence of solutions for fractional Langevin equation with infinite-point boundary conditions. J. Appl. Math. Comput. 53, 683-692 (2017)

32. Mawhin, J.: Topological Degree Methods in Nonlinear Boundary Value Problems, Expository Lectures from the CBMS Regional Conference Held at Harvey Mudd College, Claremont, Calif., June 9-15. CBMS Regional Conference Series in Mathematics. Am. Math. Soc., Providence (1979)

33. Mawhin, J: Topological degree and boundary value problems for nonlinear differential equations. In: Topological Methods for Ordinary Differential Equations, Montecatini Terme, 1991. Lecture Notes in Math., vol. 1537, pp. 74-142. Springer, Berlin (1993)

34. Bai, Z.B., Lü, H.S.: Positive solutions for boundary value problem of nonlinear fractional differential equation. J. Math. Anal. Appl. 311, 495-505 (2005)

35. Ma, R.Y., Fan, H.X., Han, X.L.: Positive solutions of nonlinear $\infty$-point boundary value problems. Acta Math. Sci. Ser. A Chin. Ed. 29, 699-706 (2009)

\section{Submit your manuscript to a SpringerOpen ${ }^{\circ}$ journal and benefit from:}

- Convenient online submission

- Rigorous peer review

- Open access: articles freely available online

- High visibility within the field

- Retaining the copyright to your article

Submit your next manuscript at $\gg$ springeropen.com 